\title{
Cost-effective laparoscopic appendicectomy
}

\section{Duxbury, N. Keeling}

Department of Surgery, West Suffolk Hospital, Bury St. Edmunds, IP33 2QZ, United Kingdom

[Surg Endosc (2006) 20: 535, DOI: 10.1007/s00464-004-2276-2]

The title of this letter to the editor was published incorrectly, and it is corrected here. 